\title{
PLANE CONSTANTLY-INCLINED MHD FLOW WITH ISOMETRIC GEOMETRY
}

\author{
$\mathrm{By}$ \\ O. P. CHANDNA AND H. TOEWS \\ University of Windsor, Windsor, Ontario
}

1. Introduction. This paper deals with plane, steady flow of a viscous, incompressible fluid of infinite electrical conductivity, the magnetic field lines meeting the streamlines at a fixed non-zero angle. In Sec. 2, a new system of flow equations governing these flows is obtained. This system is used in Secs. 3 and 4 to obtain the solutions when the streamlines and their orthogonal trajectories generate an isometric net. Chandna and Garg [1] studied the possible geometries of an isometric streamline pattern. These possibilities are also confirmed by the present work.

2. Equations of motion. The steady plane motion of a viscous incompressible fluid of infinite electrical conductivity is governed by the following system of equations:

$$
\begin{aligned}
\operatorname{div} \mathbf{v} & =0, \\
(\mathbf{v} \cdot \operatorname{grad}) \mathbf{v}+\frac{1}{\rho} \operatorname{grad} p & =\nu \nabla^{2} \mathbf{v}+\frac{\mu}{\rho}(\operatorname{curl} \mathbf{H}) \times \mathbf{H}, \\
\operatorname{curl}(\mathbf{v} \times \mathbf{H}) & =0, \\
\operatorname{div} \mathbf{H} & =0,
\end{aligned}
$$

where $\mathbf{v}$ denotes the velocity vector, $\mathbf{H}$ the magnetic field vector, $p$ the pressure, $\rho$ the constant fluid density, $\nu$ the constant kinematic viscosity and $\mu$ the constant magnetic permeability.

In this paper, we study non-aligned plane flows for which the magnetic lines lie in the flow plane and are constantly inclined to the streamlines everywhere in the flow region. Letting $\phi \neq 0$ denote the constant angle between $\mathbf{v}=\left(v_{1}, v_{2}\right), \mathbf{H}=\left(H_{1}, H_{2}\right)$ in the $(x, y)$ plane and employing (3), we find

$$
v_{1} H_{2}-v_{2} H_{1}=v H \sin \phi=A
$$

where $v$ and $H$ are the magnitudes of velocity and magnetic intensity vectors respectively and $A$ is an arbitrary constant which is non-zero due to the exclusion of aligned flows. Since $\phi$ is constant, Eq. (5) implies the existence of a constant $B$ so that

$$
v_{1} H_{1}+v_{2} H_{2}=v H \cos \phi=B
$$

where

$$
B=A \cot \phi, \quad v^{2} H^{2}=A^{2}+B^{2}=A^{2} \operatorname{cosec}^{2} \phi .
$$

The constant $B$ is zero if and only if the flow is a crossed flow when $\mathbf{v}$ and $\mathbf{H}$ are mutually orthogonal.

* Received June 14, 1975. 
Solving (5) and (6) for $\mathbf{v}$ and $\mathbf{H}$, we have

$$
\begin{aligned}
\mathbf{v} & =\frac{A}{H^{2}} \mathbf{H} \times \mathbf{k}+\frac{B}{H^{2}} \mathbf{H}, \\
\mathbf{H} & =\frac{A}{v^{2}} \mathbf{k} \times \mathbf{v}+\frac{B}{v^{2}} \mathbf{v}
\end{aligned}
$$

where $\mathbf{k}$ is the unit vector normal to the plane of flow.

Employing (8) in (1) and using (4), we have

$$
\mathbf{k} \cdot \operatorname{curl} \mathbf{H}=2 \mathbf{k} \cdot(\operatorname{grad} \ln H \times \mathbf{H})+\frac{2 B}{A} \operatorname{grad} \ln H \cdot \mathbf{H} .
$$

This equation, using (7) and (8), yields that the current density vector $\mathbf{J}=\operatorname{curl} \mathbf{H}$ is given by

$$
\operatorname{curl} \mathbf{H}=\left\{\frac{B^{2}+A^{2}}{A} \operatorname{grad}\left(\frac{1}{v^{2}}\right) \cdot \mathbf{v}\right\} \mathbf{k} .
$$

Using (9) and (10) in (2), we find for the linear momenutm equation the form

$$
(\mathbf{v} \cdot \operatorname{grad}) \mathbf{v}+\operatorname{grad}\left(\frac{p}{\rho}\right)=\nu \nabla^{2} \mathbf{v}+\frac{\mu}{\rho}\left\{\frac{B^{2}+A^{2}}{2 A} \operatorname{grad}\left(\frac{1}{v^{4}}\right) \cdot \mathbf{v}\right\}\{B \mathbf{k} \times \mathbf{v}-A \mathbf{v}\} .
$$

Employing (9) in (4) and using (1), we find that $\mathbf{v}$ satisfies

$$
\left\{\frac{2}{A} \operatorname{grad} \ln v \cdot(A \mathbf{k} \times \mathbf{v}+B \mathbf{v})\right\} \mathbf{k}+\operatorname{curl} \mathbf{v}=0 .
$$

Therefore, we have the following:

The system of equations (1)-(4) governing the steady plane motion of a viscous incompressible fluid of infinite electrical conductivity is replaced by the system of equations (1), (9), (11) and (12) for constantly inclined non-aligned flows.

Eqs. (1) and (12) are two equations in $\mathbf{v}$ and can be employed to solve for the velocity field. However, the solution thus obtained must satisfy the integrability condition for the pressure function which is derived by taking curl of (11). Having obtained $\mathbf{v}$, we solve for the pressure function and the magnetic field by employing (11) and (9) respectively.

3. Solutions of flows having an isometric streamline pattern. Let

$$
x=x(\alpha, \beta), \quad y=y(\alpha, \beta)
$$

define a system of orthogonal curvilinear coordinates in the plane of flow such that the curves $\beta(x, y)=$ const. represent the streamlines and $\alpha(x, y)=$ const. represent their orthogonal trajectories. Letting $e_{1}$ be the unit tangent vector to $\beta=$ const. in the direction of increasing $\alpha, \mathbf{e}_{2}$ the unit tangent vector to $\alpha=$ const., $h_{1}(\alpha, \beta) d \alpha$ and $h_{2}(\alpha, \beta) d \beta$ the components of a vector elements of arc length, we have

$$
\begin{aligned}
\mathbf{v} & =v(\alpha, \beta) \mathbf{e}_{1}, \\
d s^{2} & =h_{1}{ }^{2}(\alpha, \beta) d \alpha^{2}+h_{2}{ }^{2}(\alpha, \beta) d \beta^{2} .
\end{aligned}
$$

In the present section we enquire what possible solutions for constantly inclined non- 
aligned flows are possible when flow streamlines and their orthogonal trajectories form an isometric net. Therefore, we search for solutions when metric coefficients of the natural, i.e. streamline, coordinates satisfy the condition

$$
h_{1}{ }^{2}(\alpha, \beta)=h_{2}{ }^{2}(\alpha, \beta)=h(\alpha, \beta) \quad \text { (say), }
$$

where

$$
h(\alpha, \beta)=\left(\frac{\partial x}{\partial \alpha}\right)^{2}+\left(\frac{\partial y}{\partial \alpha}\right)^{2}=\left(\frac{\partial x}{\partial \beta}\right)^{2}+\left(\frac{\partial y}{\partial \beta}\right)^{2}
$$

and $h(\alpha, \beta)$ satisfies the Gauss's equation [2]

$$
\left(\frac{\partial^{2}}{\partial \alpha^{2}}+\frac{\partial^{2}}{\partial \beta^{2}}\right) \ln \sqrt{ } h=0 .
$$

For our analysis, we use some properties of an isometric orthogonal net and these are stated in the following lemma [2]:

Lemma: If $f(z)=\alpha(x, y)+i \beta(x, y)$ is a complex function so that the curves $\alpha=$ const. and $\beta=$ const. generate an orthogonal isometric net, then $f(z)=\alpha(x, y)+i \beta(x, y)$, $z(\xi)=x(\alpha, \beta)+i y(\alpha, \beta)$ are analytic functions of $z=x+i y, \xi=\alpha+i \beta$ respectively and, furthermore,

$$
\begin{gathered}
\frac{f^{\prime \prime}(z)}{\left\{f^{\prime}(z)\right\}^{2}}=-\frac{1}{2 h} \frac{\partial h}{\partial \alpha}+i \frac{1}{2 h} \frac{\partial h}{\partial \beta} \\
\frac{\partial W_{1}}{\partial \alpha}=\frac{\partial W_{2}}{\partial \beta}, \quad \frac{\partial W_{2}}{\partial \alpha}=-\frac{\partial W_{1}}{\partial \beta}
\end{gathered}
$$

where

$$
W_{1}=-\frac{1}{2 h} \frac{\partial h}{\partial \alpha}, \quad W_{2}=\frac{1}{2 h} \frac{\partial h}{\partial \beta}
$$

and $h(\alpha, \beta)$ is defined by (16).

Now, returning to our constantly inclined non-aligned flows governed by the system of equations (1), (11), (12) and (9), we find that, relative to the chosen orthogonal natural isometric net, the flow is ogverned by:

$$
\begin{aligned}
& \frac{\partial}{\partial \alpha}(\sqrt{ } h v)=0, \\
& v \frac{\partial v}{\partial \alpha}+\frac{1}{\rho} \frac{\partial p}{\partial \alpha}=\nu \frac{\partial}{\partial \beta}\left\{\frac{1}{h} \frac{\partial}{\partial \beta}(\sqrt{ } h v)\right\}+\frac{2 \mu\left(A^{2}+B^{2}\right)}{\rho v^{3}} \frac{\partial v}{\partial \alpha}, \\
& \left.\frac{v}{\sqrt{ } h} \frac{\partial}{\partial \beta}(\sqrt{ } h v)-v \frac{\partial v}{\partial \beta}-\frac{1}{\rho} \frac{\partial p}{\partial \beta}=\nu \frac{\partial}{\partial \alpha}\left\{\frac{1}{h} \frac{\partial}{\partial \beta} \sqrt{ } h v\right)\right\}+\frac{2 \mu B\left(A^{2}+B^{2}\right)}{A \rho v^{3}} \frac{\partial v}{\partial \alpha}, \\
& B \frac{\partial v}{\partial \alpha}+A \frac{\partial v}{\partial \beta}=\frac{1}{2 \sqrt{ } h} \frac{\partial}{\partial \beta}(\sqrt{ } h v), \\
& \mathbf{H}=\frac{B}{v} \mathbf{e}_{1}+\frac{A}{v} \overline{\mathbf{e}}_{2} .
\end{aligned}
$$


It follows from (20) that

$$
v(\alpha, \beta)=\frac{\psi(\beta)}{\sqrt{ } h}
$$

where $\psi(\beta)$ is an arbitrary differentiable function of $\beta$.

Employing (25) in (23), we obtain

$$
A W_{2}-B W_{1}=A \frac{\psi^{\prime}(\beta)}{2 \psi(\beta)}
$$

where $W_{1}(\alpha, \beta)$ and $W_{2}(\alpha, \beta)$ are defined in (19). Differentiating (26) with respect to $\alpha$ and using the Cauchy-Riemann conditions satisfied by $W_{1}(\alpha, \beta), W_{2}(\alpha, \beta)$ as given in (19), we obtain the following first-order uncoupled partial differential equations:

$$
\begin{aligned}
& A \frac{\partial W_{1}}{\partial \beta}+B \frac{\partial W_{1}}{\partial \alpha}=0 \\
& B \frac{\partial W_{2}}{\partial \beta}-A \frac{\partial W_{2}}{\partial \alpha}=0 .
\end{aligned}
$$

Solving (27) and (28), we obtain the general solutions for $W_{1}$ and $W_{2}$

$$
\begin{aligned}
& W_{1}(\alpha, \beta)=f(A \alpha-B \beta), \\
& W_{2}(\alpha, \beta)=g(A \beta+B \alpha) .
\end{aligned}
$$

Here $f$ and $g$ are arbitrary functions of their arguments. If we define $\xi=A \alpha-B \beta$ and $\eta=A \beta+B \alpha$, it follows that the transformation Jacobian from the $(\alpha, \beta)$-plane to the $(\xi, \eta)$-plane is $|\partial(\xi, \eta) / \partial(\alpha, \beta)|=A^{2}+B^{2} \neq 0$, Therefore we can regard $\xi$ and $\eta$ as two independent variables. Substituting (29) in (19) and taking $\xi, \eta$ as the independent variables, we find that $W_{1}^{\prime}(\xi)=W_{2}^{\prime}(\eta)$. Therefore, we have

$$
W_{1}=C(A \alpha-B \beta)+C_{1}, \quad W_{2}=C(A \beta+B \alpha)+C_{2}
$$

where $C, C_{1}$ and $C_{2}$ are arbitrary constants.

Employing (30) in (19) and (26), we obtain

$$
\begin{aligned}
& \frac{\partial}{\partial \alpha}(\ln h)=2 C(B \beta-A \alpha)-2 C_{1}, \\
& \frac{\partial}{\partial \beta}(\ln h)=2 C(A \beta+B \alpha)+2 C_{2}, \\
& \frac{d}{d \beta}(\ln \psi)=\frac{2 C}{A}\left(A^{2}+B^{2}\right) \beta-\frac{2 B C_{1}}{A}+2 C_{2} .
\end{aligned}
$$

Integration of (31) yields

$$
\begin{aligned}
h(\alpha, \beta) & =\exp \left\{A C\left(\beta^{2}-\alpha^{2}\right)+2 C B \alpha \beta+2 C_{2} \beta-2 C_{1} \alpha+C_{3}\right\}, \\
\psi(\beta) & =\exp \left\{\frac{C}{A}\left(A^{2}+B^{2}\right) \beta^{2}+\left(2 C_{2}-\frac{2 B C_{1}}{A}\right) \beta+C_{4}\right\},
\end{aligned}
$$

where $C_{3}$ and $C_{4}$ are arbitrary constants. Therefore, for an isometric streamline pattern, solutions for $v(\alpha, \beta)$ and $h(\alpha, \beta)$ satisfying (20) and (23) are given by (25) and (32) with 
$\psi(\beta)$ given by (33). However, these solutions must satisfy the integrability equation for $p(\alpha, \beta)$ obtained from (21), (22) and given by

$$
\begin{aligned}
\frac{\partial}{\partial \alpha}\left\{\frac{v}{\sqrt{ } h} \frac{\partial}{\partial \beta}(\sqrt{ } h v)\right\}-\nu\left(\frac{\partial^{2}}{\partial \alpha^{2}}\right. & \left.+\frac{\partial^{2}}{\partial \beta^{2}}\right)\left\{\frac{1}{h} \frac{\partial}{\partial \beta}(\sqrt{ } h v)\right\} \\
& -\frac{2 \mu}{\rho}\left(A^{2}+B^{2}\right)\left\{\frac{\partial}{\partial \beta}\left(\frac{1}{v^{3}} \frac{\partial v}{\partial \alpha}\right)+\frac{B}{A} \frac{\partial}{\partial \alpha}\left(\frac{1}{v^{3}} \frac{\partial v}{\partial \alpha}\right)\right\}=0 .
\end{aligned}
$$

Eliminating $v(\alpha, \beta)$ between (25) and (34), we find that $\psi(\beta)$ and $h(\alpha, \beta)$ must satisfy

$$
\begin{gathered}
\psi \psi^{\prime}\left\{\frac{1}{h} \frac{\partial h}{\partial \alpha}\right\}+\nu\left[\psi^{\prime \prime \prime}-2 \psi^{\prime \prime}\left\{\frac{1}{h} \frac{\partial h}{\partial \beta}\right\}+\psi^{\prime}\left\{2\left(\frac{1}{h} \frac{\partial h}{\partial \alpha}\right)^{2}+2\left(\frac{1}{h} \frac{\partial h}{\partial \beta}\right)^{2}-\frac{1}{h} \frac{\partial^{2} h}{\partial \alpha^{2}}-\frac{1}{h} \frac{\partial^{2} h}{\partial \beta^{2}}\right\}\right] \\
-\frac{\mu\left(A^{2}+B^{2}\right)}{2 A \rho} \cdot \frac{h^{2}}{\psi^{2}}\left\{\frac{2 A}{h} \frac{\partial^{2} h}{\partial \alpha \partial \beta}+\frac{2 B}{h} \frac{\partial^{2} h}{\partial \alpha^{2}}-\frac{4 A}{h} \frac{\partial h}{\partial \alpha} \frac{\psi^{\prime}}{\psi}\right\}=0 .
\end{gathered}
$$

Employing (32) in (35), we get

$$
\begin{aligned}
\psi \psi^{\prime}(2 C B \beta & \left.-2 A C \alpha-2 C_{1}\right)+\nu \psi^{\prime \prime \prime}-2 \nu \psi^{\prime \prime}\left(2 A C \beta+2 B C \alpha+2 C_{2}\right) \\
& +\nu \psi^{\prime}\left\{\left(2 C B \beta-2 A C \alpha-2 C_{1}\right)^{2}+\left(2 A C \beta+2 B C \alpha+2 C_{2}\right)^{2}\right\} \\
& -\frac{4 \mu\left(A^{2}+B^{2}\right)}{A \rho \psi^{2}}\left\{h^{2}\left(B C \beta-A C \alpha-C_{1}\right)\left(A^{2} C \beta+B^{2} C \beta+A C_{2}-B C_{1}\right)\right. \\
& \left.-\frac{A \psi^{\prime}}{\psi} h^{2}\left(B C \beta-A C \alpha-C_{1}\right)\right\}=0 .
\end{aligned}
$$

Differentiating (36) thrice with respect to $\alpha$, we obtain

$$
\begin{aligned}
\frac{\partial^{3}}{\partial \alpha^{3}}\left[h^{2}\left(B C \beta-A C \alpha-C_{1}\right)\left(A^{2} C \beta+B^{2} C \beta+A C_{2}-B C_{1}\right)\right] & \\
& -\frac{A \psi^{\prime}}{\psi} \frac{\partial^{3}}{\partial \alpha^{3}}\left[h^{2}\left(B C \beta-A C \alpha-C_{1}\right)\right]=0 .
\end{aligned}
$$

Using (32), (33) in (37) and simplifying, we have

$$
\begin{aligned}
\left\{C\left(A^{2}+B^{2}\right) \beta+A C_{2}-B C_{1}\right\} & \left\{16\left(B C \beta-A C \alpha-C_{1}\right)^{4}\right. \\
& \left.-24 A C\left(B C \beta-A C \alpha-C_{1}\right)^{2}+3 A^{2} C^{2}\right\}=0 .
\end{aligned}
$$

Eq. (38) is of fifth degree in $\alpha, \beta$ and is satisfied throughout the flow region. This requires that all its coefficients must be zero. In particular, equating the coefficient of $\alpha^{4} \beta$ to zero, we have

$$
A^{4} C^{5}\left(A^{2}+B^{2}\right)=0 .
$$

Since $A \neq 0$ and (39) must hold true, we find that $C=0$. Setting $C=0$ in (38), it follows that for our flows the arbitrary constants $C_{1}$ and $C_{2}$ must satisfy

$$
C_{1}\left(A C_{2}-B C_{1}\right)=0
$$

Summing up the above results, we have:

Theorem: If the natural net is isometric in a steady incompressible viscous prefectly conducting constantly-inclined non-aligned plane flow, then the metric of this net, 
the flow speed and the magnetic intensity are given by

$$
\begin{gathered}
h(\alpha, \beta)=\exp \left\{2 C_{2} \beta-2 C_{1} \alpha+C_{3}\right\} \\
v(\alpha, \beta)=\exp \left(C_{4}-\frac{1}{2} C_{3}\right) \exp \left\{\left(C_{2}-\frac{2 B C_{1}}{A}\right) \beta+C_{1} \alpha\right\}, \\
\mathbf{H}(\alpha, \beta)=\left[\exp \left(\frac{1}{2} C_{3}-C_{4}\right) \exp \left\{\left(\frac{2 B C_{1}}{A}-C_{2}\right) \beta-C_{1} \alpha\right\}\right]\left(B \mathbf{e}_{1}+A \mathbf{e}_{2}\right),
\end{gathered}
$$

wherein the arbitrary constants $C_{1}$ end $C_{2}$ must satisfy (40).

4. Classification and geometry of isometric flows. For flows with isometric streamline pattern, $C=0$ and $C_{1}, C_{2}$ must satisfy (40). Therefore, this allows one of the following three possibilities:

$$
\begin{aligned}
& C=0, \quad C_{1} \neq 0 \quad \text { and } C_{2}=\frac{B}{A} C_{1} ; \\
& C=0, \quad C_{1}=0 \quad \text { and } C_{2} \neq 0 ; \\
& C=C_{1}=C_{2}=0 .
\end{aligned}
$$

Employing (31) in (18) and taking $C=0$, which is true for all possibilities, we have

$$
\frac{f^{\prime \prime}(z)}{\left\{f^{\prime}(z)\right\}^{2}}=C_{1}+i C_{2} \text {. }
$$

For possibilities (i) and (ii), $C_{1}+i C_{2} \neq 0$, and integration of (42) yields

$$
f(z)=-\frac{1}{\left(C_{1}+i C_{2}\right)} \ln (z-D)+E
$$

where $D=D_{1}+i D_{2}$ and $E=E_{1}+i E_{2}$ are two arbitrary complex constants.

Letting $z-D=r \exp (i \theta)$, where $(r, \theta)$ are polar coordinates, and recalling that $f(z)=\alpha+i \beta$, separation of real and imaginary parts of (43) yields

$$
\begin{aligned}
& \alpha(r, \theta)=-\frac{1}{\left(C_{1}^{2}+C_{2}^{2}\right)}\left\{C_{1}\left(\ln r+E_{1}\right)+C_{2}\left(\theta+E_{2}\right)\right\}, \\
& \beta(r, \theta)=\frac{1}{\left(C_{1}^{2}+C_{2}^{2}\right)}\left\{C_{2}\left(\ln r+E_{1}\right)-C_{1}\left(\theta+E_{2}\right)\right\} .
\end{aligned}
$$

For possibility (iii), $C_{1}=C_{2}=0$, integration of (42) and separation into real and imaginary parts yields

$$
\begin{aligned}
& \alpha(x, y)=L_{1} x-L_{2} y+M_{1}, \\
& \beta(x, y)=L_{2} x+L_{1} y+M_{2},
\end{aligned}
$$

where $L_{1}, L_{2}, M_{1}$ and $M_{2}$ are real arbitrary constants. Summing up, we take the above possibilities one by one with solutions and geometries.

Type (i): Taking $C_{1} \neq 0$ and $C_{2}=(B / A) C_{1}$ in (44), the streamlines are given by Bln $r-A \theta=$ constant. These are logarithmic spirals. The flow speed, the magnetic intensity and the metric of the net so generated are given by (41) where $C_{2}=(B / A) C_{1}$. 
Using these in (21), (22) and integrating, we find

$$
\begin{aligned}
p=-\frac{\rho}{2} \exp \left\{2 C_{1}\left(\alpha-\frac{B}{A} \beta\right)+2 C_{4}-C_{3}\right\} & \\
& -\mu\left(A^{2}+B^{2}\right) \exp \left\{2 C_{1}\left(\frac{B}{A} \beta-\alpha\right)+C_{3}-2 C_{4}\right\}+C_{5}
\end{aligned}
$$

where $C_{5}$ is an arbitrary constant.

Type (ii): Taking $C_{1}=0$ and $C_{2} \neq 0$ in (44), we find that the streamlines are given by $\ln r=$ constant. Therefore, the streamlines in this case are a family of concentric circles. Solutions are given by (41) with $C_{1}=0$ and the pressure function is

$$
p=\frac{\rho}{2} \exp \left\{2\left(C_{2} \beta+C_{4}-C_{3}\right)\right\}+C_{6}
$$

where $C_{6}$ is an arbitrary constant.

Type (iii): For this type of flows, the streamlines are given by $L_{2} x+L_{1} y=$ constant. These are a family of parallel straight lines. The solutions of these flow are given by (41) and (47) with $C_{1}=C_{2}=0$.

The discussion carried out in this paper holds true for crossed flows when $B=0$. However, the streamline geometry in flows of type $(i)$ will change, with a family of concurrent straight streamlines replacing logarithmic spirals.

\section{REFERENCES}

[1] O. P. Chandna and M. R. Garg, The flow of a viscous MHD fluid, Quart. Appl. Math. 34, 287(1976)

[2] Berker, R., Integration des équations de mouvement d'un fluide visques incompressible, Vol. VIII/2 Strömungsmechanik II, Handbuch der Physik, Berlin (1963) 